\title{
Physical Activity Targeted at Maximal Lipid Oxidation: A Meta-Analysis
}

\author{
A. J. Romain, ${ }^{1,2}$ M. Carayol, ${ }^{1}$ M. Desplan, ${ }^{3}$ C. Fedou, ${ }^{3,4}$ G. ${ }^{2}$ inot, ${ }^{1}$ J. Mercier, ${ }^{3}$ A. Avignon, ${ }^{2,3}$ \\ and J. F. Brun ${ }^{3,4}$ \\ ${ }^{1}$ EA 4556 Epsylon: Laboratory of Dynamics of human Abilities \& Health Behaviors, University Montpellier 1, \\ 34000 Montpellier, France \\ ${ }^{2}$ Department of Nutrition and Diabetes, University Hospital of Montpellier, 34295 Montpellier, France \\ ${ }^{3}$ INSERM U1046 "Physiologie et Médecine Expérimentale du Cour et du Muscle”, Université Montpellier 1, Université Montpellier 2 \\ et Centre Hospitalier Universitaire Lapeyronie, 34295 Montpellier, France \\ ${ }^{4}$ Department of Clinical Physiology (CERAMM), University Hospital of Montpellier, 34295 Montpellier, France
}

Correspondence should be addressed to J. F. Brun, j-brun@chu-montpellier.fr

Received 7 January 2012; Revised 26 March 2012; Accepted 22 May 2012

Academic Editor: Jørn Helge

Copyright (C) 2012 A. J. Romain et al. This is an open access article distributed under the Creative Commons Attribution License, which permits unrestricted use, distribution, and reproduction in any medium, provided the original work is properly cited.

\begin{abstract}
Exercise is recognized as a part of the management of obesity and diabetes. Various protocols of exercise are proposed for the management of obesity, diabetes, and other metabolic diseases. One of the strategies proposed by several authors is low intensity endurance training targeted at the level of maximal oxidation. Large series using this technique are lacking. Addressing this issue, we performed a meta-analysis of the studies on anthropometric measurements. From a database of 433 articles, 15 were selected, including 279 subjects with 6 different populations. Studies duration ranged from 2 months to 12 months. Concerning weight loss, in the intervention versus control analysis, five studies with 185 participants were included with a significant effect size favors exercise $(P=0.02)$ without significant heterogeneity $\left(I^{2}=0.0 \%, P=0.83\right)$. Further randomized controlled trials for comparing it with other exercise protocols and defining its dose effectiveness on large samples are needed.
\end{abstract}

\section{Introduction}

Exercise training is now widely recognized as a key component of the management of obesity $[1,2]$ and diabetes [3]. At the beginning of the 21st century, the description of a curve of lipid oxidation $[4,5]$ led to the hypothesis that endurance exercise in obesity and diabetes should be targeted at this level of maximal lipid oxidation in order to obtain an optimal effect on lipids [6]. This curve is derived from the crossover concept [7] which is a physiological theory of exercise explaining that at rest, as at low intensities of exercise, the substrate of energy that is preferentially oxidized is lipids whereas at the highest intensities, carbohydrates are preferentially oxidized. However, only a few studies have addressed this working hypothesis on exercise and obesity or diabetes, and the bulk of current literature does not take into account this concept $[8,9]$.

Despite the fact that most of these studies involve a little number of subjects and are performed over a short duration of time, it was interesting to review them in a meta-analysis. The aim of this meta-analysis was to give an overall picture of the effects of exercise training targeted on maximal lipid oxidation.

\section{Material and Methods}

2.1. Literature Search. We conducted a research on (1) Pubmed (2) ISI Web of Science from 1994 to 2012, and (3) we also manually searched articles on sciences direct database 
published from 1994 to 2012 in English or French languages. The year 1994 was chosen being the date of the publication on the crossover concept [7]. Further information about the crossover concept is provided in a recent review $[10,11]$. We (4) also included proceedings of congress when data were available by asking values to corresponding authors. Words written to perform research were "lipoxmax," "lipoxmax AND training," "lipoxmax AND physical activity," "lipoxmax AND exercise," "fatmax," "fatmax AND training", "fatmax AND physical activity", "fatmax AND exercise", "maximal fat oxidation," "maximal fat oxidation AND training," "maximal fat oxidation AND exercise," and "maximal fat oxidation AND physical activity".

Articles were selected by three different investigators. Differences of opinion for inclusion were resolved by discussion.

2.2. Study Selection. Studies were included in the metaanalysis if they met the following criteria: (1) designed as randomized controlled trial or clinical trial, (2) maximal fatoxidation point was the training method according to the described protocol, (3) participants were males or females affected by chronic diseases without age restriction, and (4) anthropometric measurements and cholesterol were defined as outcomes. If data were duplicated in more than one publication, only the most recent publication was included in the analysis.

Studies were excluded if (1) it was on animal models, (2) the study design was cross-sectional, (3) there was no intervention, and (4) the intervention was on healthy participants.

\subsection{Extraction and Classification of the Data. Descriptive} data regarding author, year of publication, pathology, study sample characteristics, type of design, and duration of training protocol were extracted from all selected articles. Weight, waist measurement, fat mass, and serum cholesterol were the selected outcomes.

2.4. Statistical Methods and Analysis. Because of heterogeneity among included studies, the effect estimates were pooled using a random effects model with the method of DerSimonian and Laird. Firstly, pre- and postintervention mean differences (and their associated standard errors) were pooled for each outcome. Secondly, another analysis consisted of results extracted from randomized controlled trials only to obtain a pooled standardized mean difference of intervention group versus control group. The second analysis was only achieved for the weight outcome as there were less than three included studies that reported mean differences for the other outcomes.

Statistical analyses were performed by using Stata software version 10 (StataCorp. 2007. Stata Statistical Software: Release 10. College Station, TX: StataCorp LP).

Heterogeneity was tested by using Cochran's chi-square test (Q) to assess the consistency of associations as usual in meta-analysis [12]. To quantify the extent of heterogeneity of this collection of studies, we estimated the between-study variance $\left(I^{2}\right)$. This $I^{2}$ statistics describes the proportion of total variance in effect estimates due to the heterogeneity among studies; homogeneous studies should have an $I^{2}$ value of 0 .

When there were sources of heterogeneity, meta regression was computed to test whether such factors as population, intervention duration, and training type (diet and exercise + diet) had an impact on the final pooled estimates and on the heterogeneity. When moderators were significant, we split the analyses according to this moderator.

Publication bias was examined with the use of funnel plot which is a scatter plot of treatment effect against a measure of study size. The presented funnel plot includes pre- and postintervention mean differences of weight as it is the outcome for which we found the highest number of included studies.

\section{Results}

Researches using our different strategies within the different databases retrieved 433 articles. A sum of 60 articles were selected on the basis of their title and abstract, then a total of 15 articles [13-28] met the inclusion criteria and were thus included in the meta-analysis. Flow chart of the study selection is available in Figure 1. Descriptive characteristics of the studies are presented in Table 1. There were 3 randomized controlled trials, 2 controlled trials, and 10 clinical trials or random trials. A total of 279 subjects were recruited through the 15 studies. We found 6 different populations. Those populations were obese adolescents [13$15,18,21,26,27$ ] and nondiabetic obese adults [24], patients with metabolic syndrome [16, 19], patients affected by human immunodeficiency virus (HIV) [17], patients with type 2 diabetes (T2D) [20, 22, 25, 28], and patients with neuroleptic treatment [23]. The study with the highest sample is a randomized controlled trial including $63 \mathrm{~T} 2 \mathrm{D}$ [28].

Several types of protocols were identified. There were studies with only training $[13,14,16-28]$, studies with diet + training $[13,15,18,21,26]$.

Duration of studies varied from 2 months to 12 months. The study that included people affected by HIV presented the longest followup [17].

Number of training sessions per week ranged from 2 sessions of 45 minutes to 4 sessions of 90 minutes per week. Two studies $[17,23]$ did not precise the number of sessions per week. One study used an incremental protocol [24] and one an exercise protocol where the numbers of sessions were progressively reduced [14]. The whole studies used ergometer as training material during their sessions.

Concerning the nutritional intervention, the whole studies used hypocaloric diet. One studies set -500 kilocalories (Kcal) per day below the energy requirements at time of the study without precision about the repartition of nutriments [21]. Three others studies set $-500 \mathrm{Kcal}$ per day with $15 \%$ from proteins, $55 \%$ from carbohydrates, and 30\% from lipids $[13,18,26]$, and 1 study set $-300 \mathrm{Kcal}$ per day with meal composed of $15 \%$ from proteins, $55 \%$ from carbohydrates and $30 \%$ from lipids [15]. 


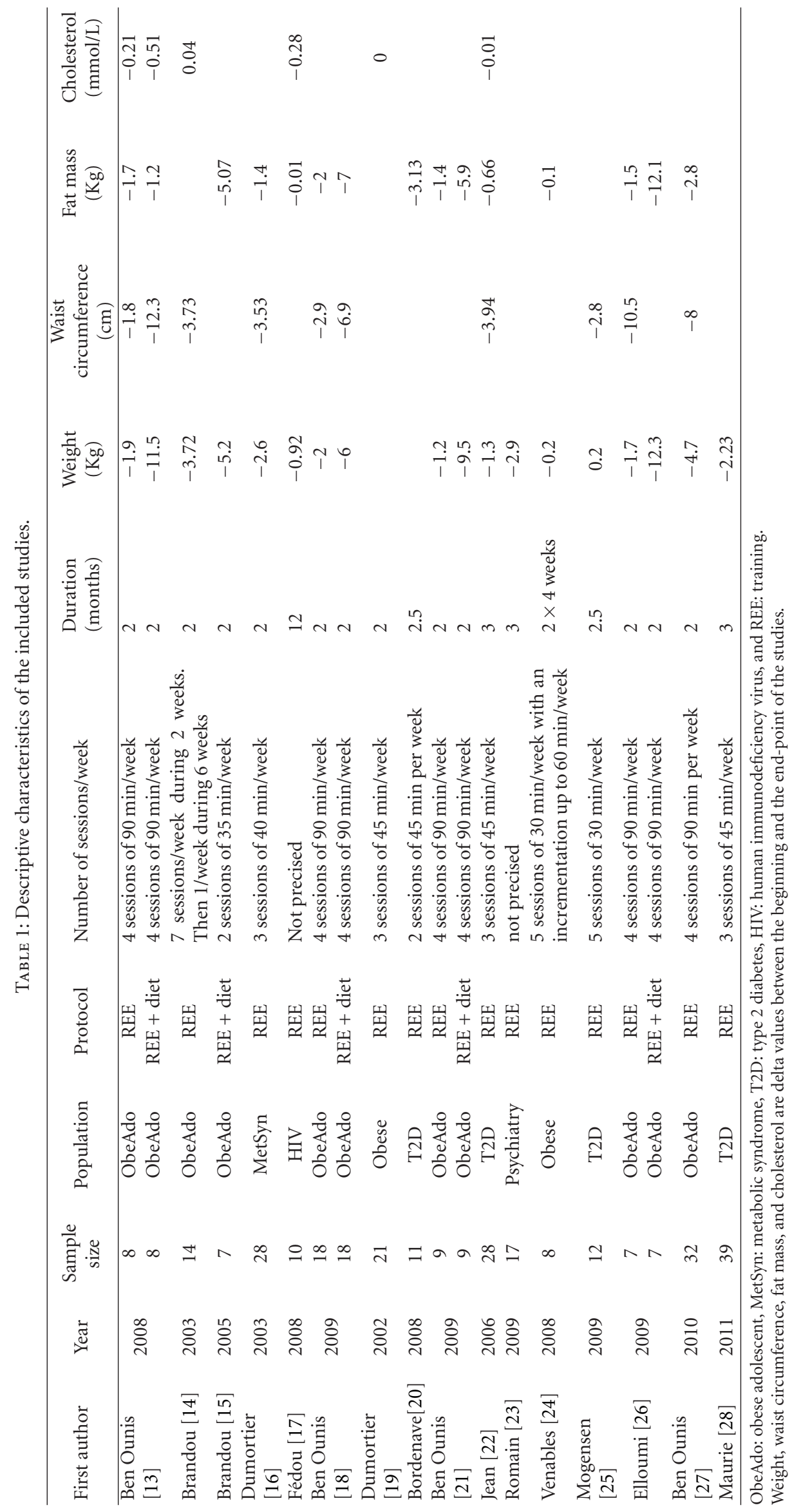




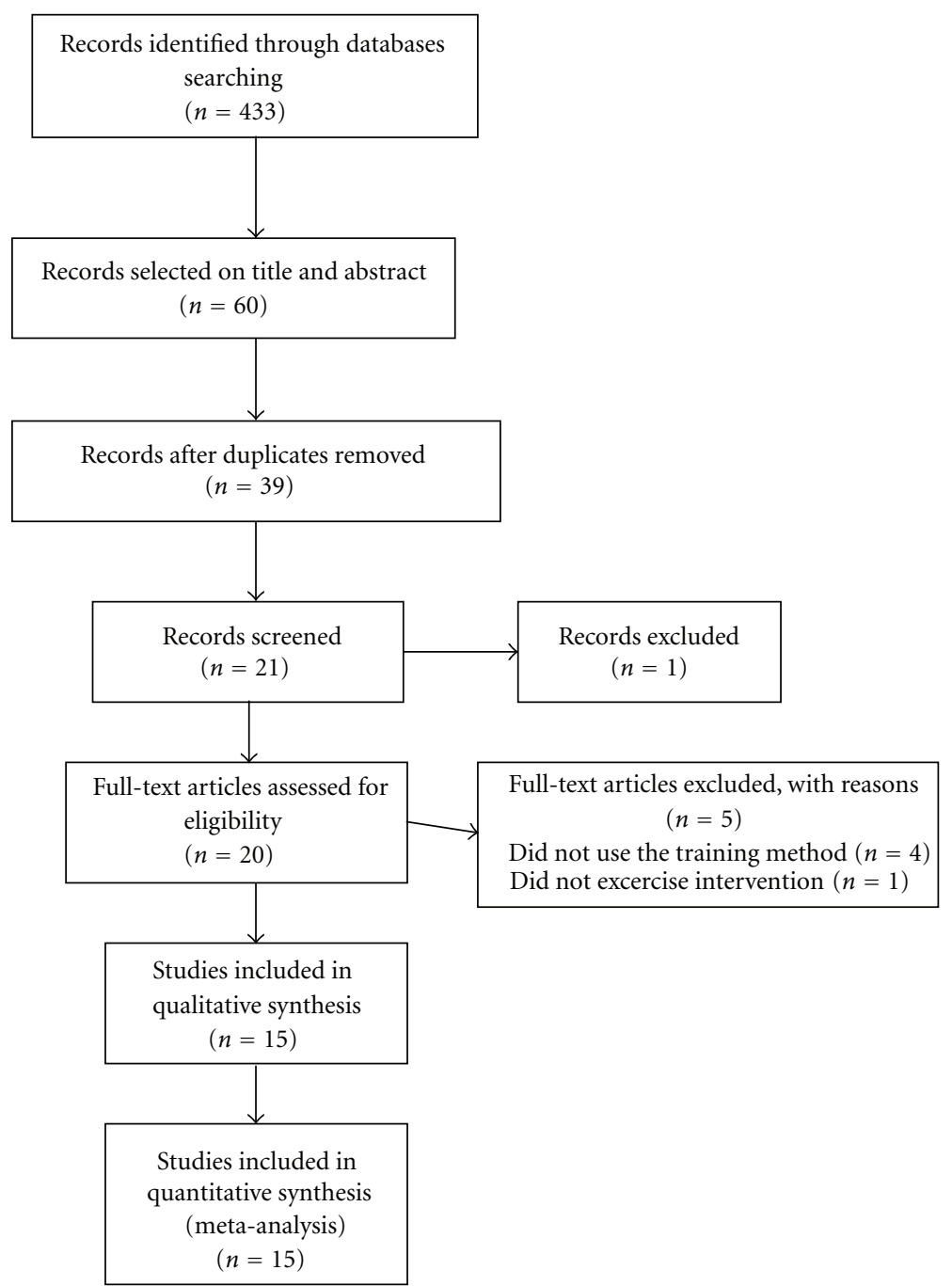

Figure 1: Flow chart of the study selection.

\subsection{Before versus after Intervention}

3.1.1. Weight. In included studies, loss of weight varied from $0 \mathrm{Kg}$ to $11.5 \mathrm{Kg}$ and 11 studies reported a significant loss between before and after intervention.

The pooled effect estimate and its associated 95\% confidence interval (CI) of weight loss of after versus before intervention was $-2.86 \mathrm{Kg}$ (95\% CI: -4.07; - 1.64) (see Figure 2). The analysis showed a significant heterogeneity among studies $\left(P<0.0001, I^{2}=82 \%\right)$.

3.1.2. Fat Mass. In included studies, loss of fat mass varied from $-0.01 \mathrm{Kg}$ to $-12.1 \mathrm{Kg}$ and 10 studies reported a significant loss between before and after intervention.

The pooled effect estimate of fat mass loss of after versus before intervention was $-4.1 \mathrm{Kg}(95 \% \mathrm{CI}:-5.8 ;-2.3)(P<$ 0.0001 ) (see Figure 3 ). The analysis showed a significant heterogeneity among studies $\left(P<0.0001, I^{2}=81 \%\right)$.
3.1.3. Waist Circumference. In included studies, changes in waist circumference varied from $-2.9 \mathrm{~cm}$ to $-12.3 \mathrm{~cm}$ and 7 studies reported a significant decrease between before and after intervention.

The pooled effect estimate of waist circumference change of after versus before intervention was $-4.9 \mathrm{~cm}(95 \% \mathrm{CI}$ : $-6.6 ;-3.2)(P<0.0001)$ (see Figure 4$)$. The analysis showed a significant heterogeneity among studies $\left(P=0.02, I^{2}=\right.$ $52 \%)$.

3.1.4. Cholesterol. In included studies, changes in total cholesterol varied from $0 \mathrm{mmol} / \mathrm{L}$ to $-0.66 \mathrm{mmol} / \mathrm{L}$ and 3 studies reported a significant change between after and after intervention. The pooled effect estimate of total cholesterol of after versus after intervention was $-0.26 \mathrm{mmol} / \mathrm{L}$ (95\% CI: $-0.35 ;-0.17)(P<0.0001)$. The analysis did not show any significant heterogeneity $(P=0.18)$. 


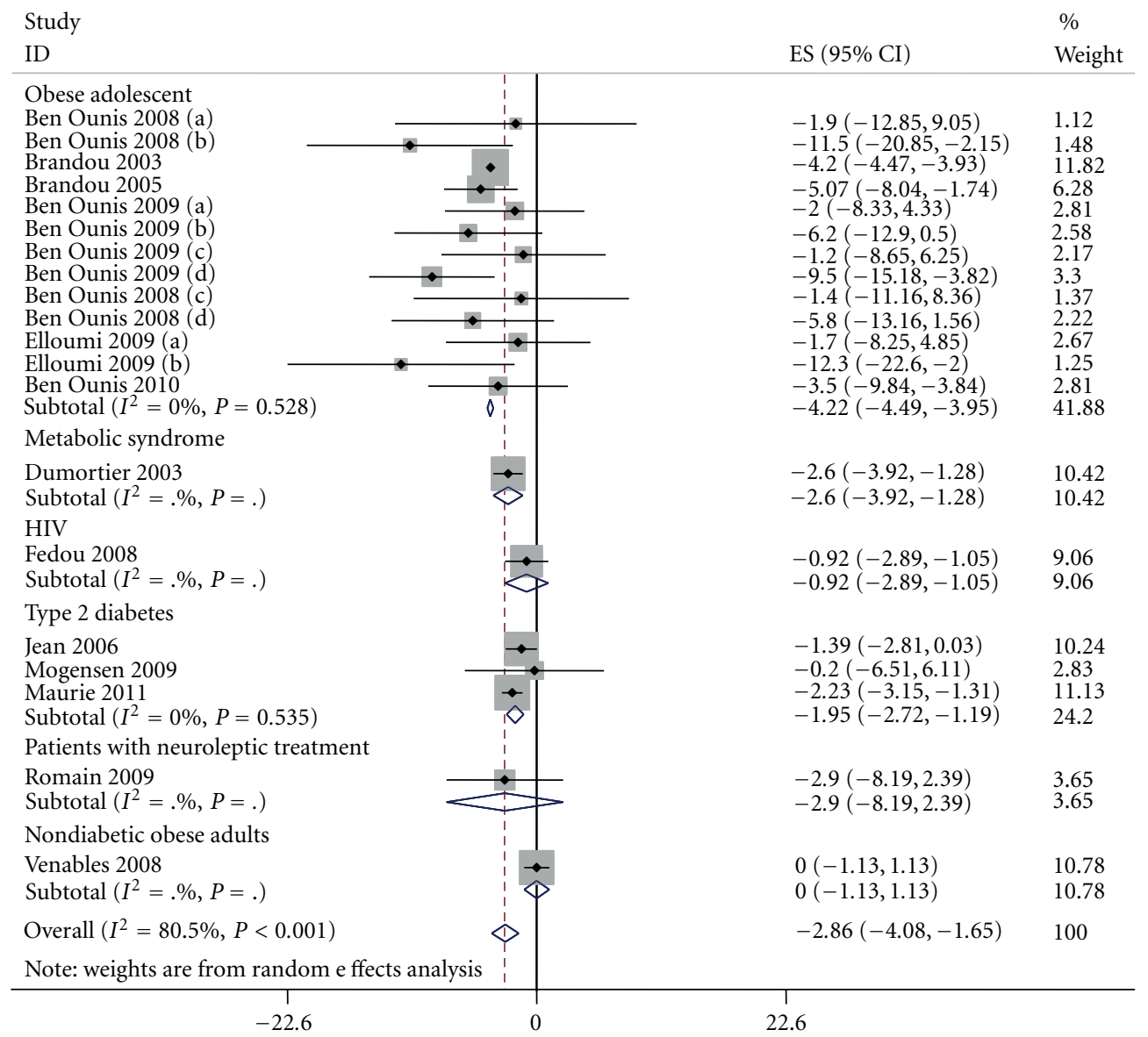

FIGURE 2: Pooled standard errors and confidence intervals of weight loss in before to after design.

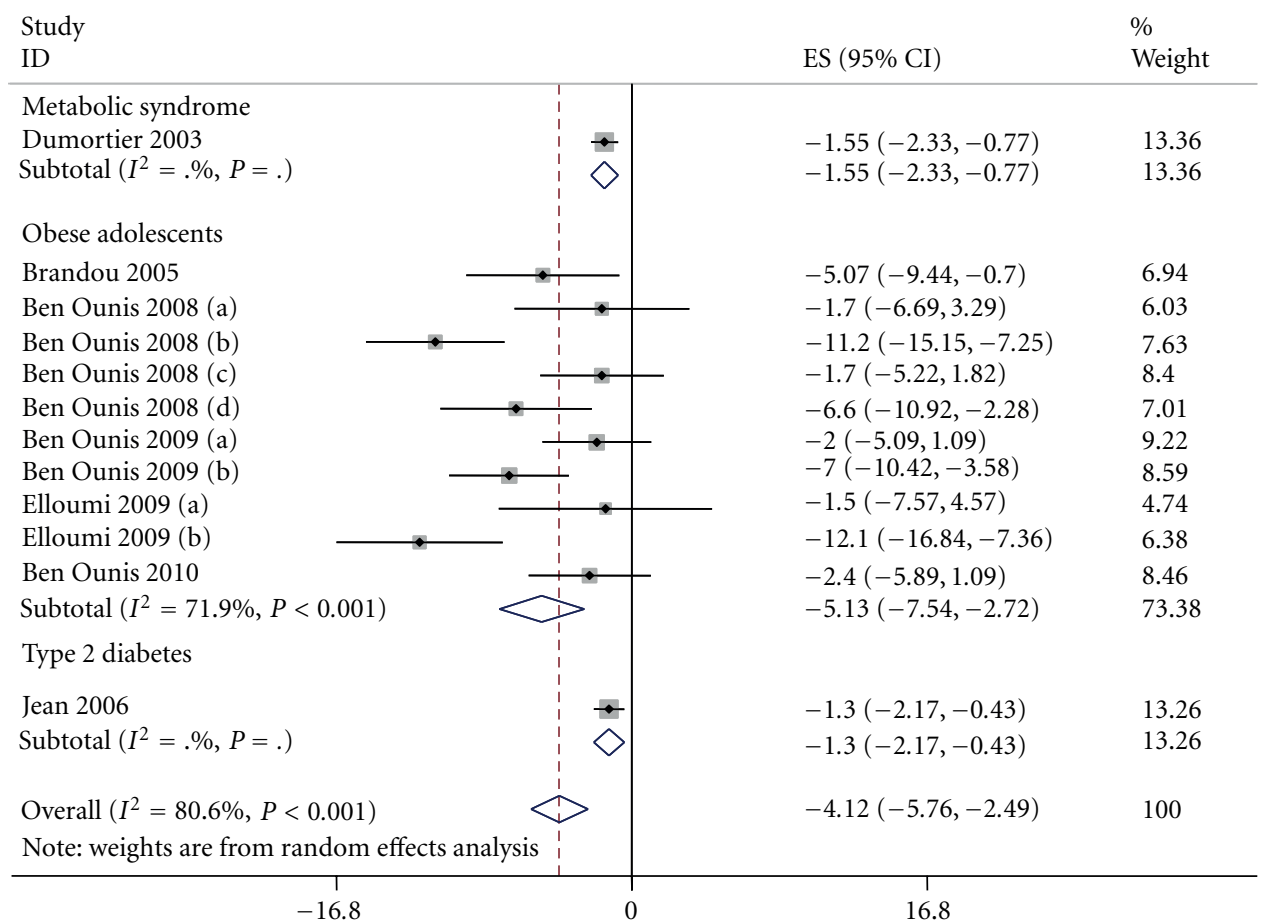

Figure 3: Pooled standard errors and confidence intervals of fat mass loss in before to after design. 


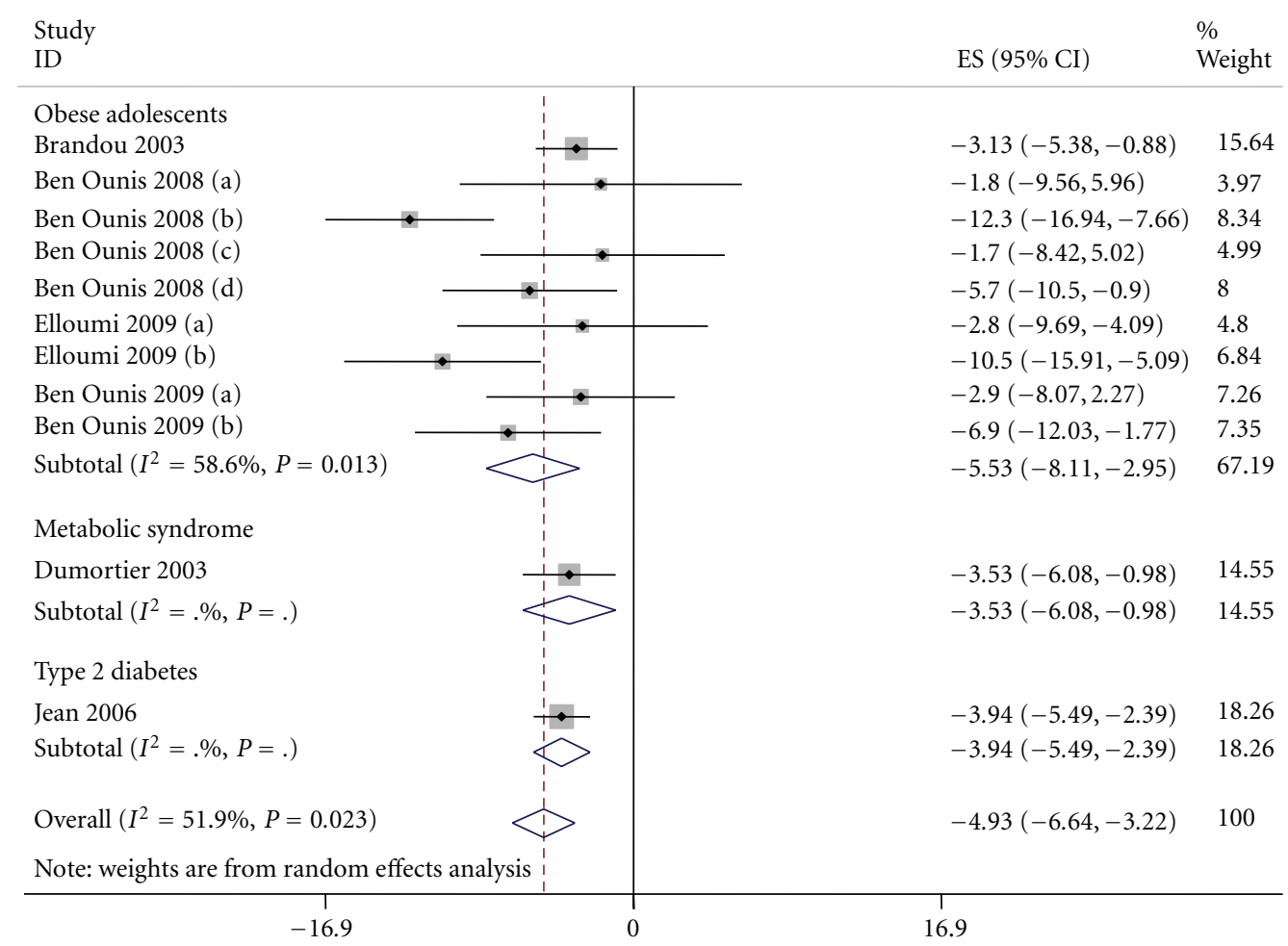

Figure 4: Pooled standard errors and confidence intervals of waist circumference in before to after design.

3.2. Intervention versus Control. Due to lack of data for fat mass, waist circumference and cholesterol, intervention versus control analysis was only computed for weight.

5 studies which involved 185 participants (128 in the intervention group and 57 in the control group) were included in the analysis. All 5 studies reported significant loss of weight in intervention group compared to control group.

The pooled standardized mean difference of intervention versus control was -0.37 (95\% CI: $-0.69 ;-0.06)(P=0.02)$ and favors intervention (see Figure 5). The analysis did not show significant heterogeneity $\left(P=0.83, I^{2}=0.0 \%\right)$.

3.3. Analyses of Moderators. All results concerning the moderators are in Table 2.

3.3.1. Weight. Concerning weight loss, the metaregression showed that population $(P<0.001)$ and nutrition intervention $(P<0.01)$ were significant moderators of weight loss while duration of intervention was not $(P=0.227)$.

As population was a significant moderator, we thus split the results according to population (see Figure 2). These secondary analyses showed that results remained significant in obese adolescent $(-4.21(95 \% \mathrm{CI}:-4.48 ;-3.94))$ with no heterogeneity $\left(P=0.52, I^{2}=0.0 \%\right)$, metabolic syndrome $(-2.60(95 \% \mathrm{CI}:-3.92 ;-1.27))$, and heterogeneity was not applicable due to the number of included studies $(n=1)$, T2D $(-1.95(95 \% \mathrm{CI}:-2.72 ;-1.18))$ with no heterogeneity $\left(P=0.53 ; I^{2}=0.0 \%\right)$.
TABLE 2: Results from analyses of moderators.

\begin{tabular}{lcccc}
\hline Moderators & Beta & Standard error & $P$ value & $95 \%$ CI \\
\hline Weight & & & & \\
$\quad$ Population & 1.05 & 0.12 & $<0.001$ & $0.81 ; 1.29$ \\
$\quad$ Duration & 0.25 & 0.20 & 0.23 & $-0.17 ; 0.67$ \\
$\quad$ Nutrition & -5.09 & 1.47 & 0.003 & $-8.18 ;-2.01$ \\
Fat mass & & & & \\
$\quad$ Population & 2.19 & 1.57 & 0.19 & $-1.32 ; 5.70$ \\
$\quad$ Duration & 1.79 & 2.93 & 0.55 & $-4.74 ; 8.34$ \\
$\quad$ Nutrition & -6.75 & 0.97 & $<0.001$ & $-8.92 ;-4.58$ \\
Waist circumference & & & & \\
$\quad$ Population & 0.95 & 1.38 & 0.51 & $-2.80 ; 4.90$ \\
$\quad$ Duration & 2.07 & 2.16 & 0.36 & $-2.80 ; 6.96$ \\
$\quad$ Nutrition & -5.37 & 1.38 & $<0.001$ & $-8.49 ;-2.25$ \\
\hline
\end{tabular}

95\% CI: 95\% confidence intervals.

Splitting the results according to an intervention in nutrition or not, the mean difference remained significant for intervention only with exercise $(-1.95$ (95\% CI: -3.28 ; $-0.62)$ ) and was larger with interventions including diet + exercise $(-6.81$ (95\% CI: $-9.15 ;-4.47))$, without overlap between the CI showing a significant difference between the two interventions. The heterogeneity remained present for intervention without nutrition $\left(P<0.001, I^{2}=85 \%\right)$ but not in those included both diet and exercise $\left(P=0.54, I^{2}=\right.$ $0.0 \%)$. 


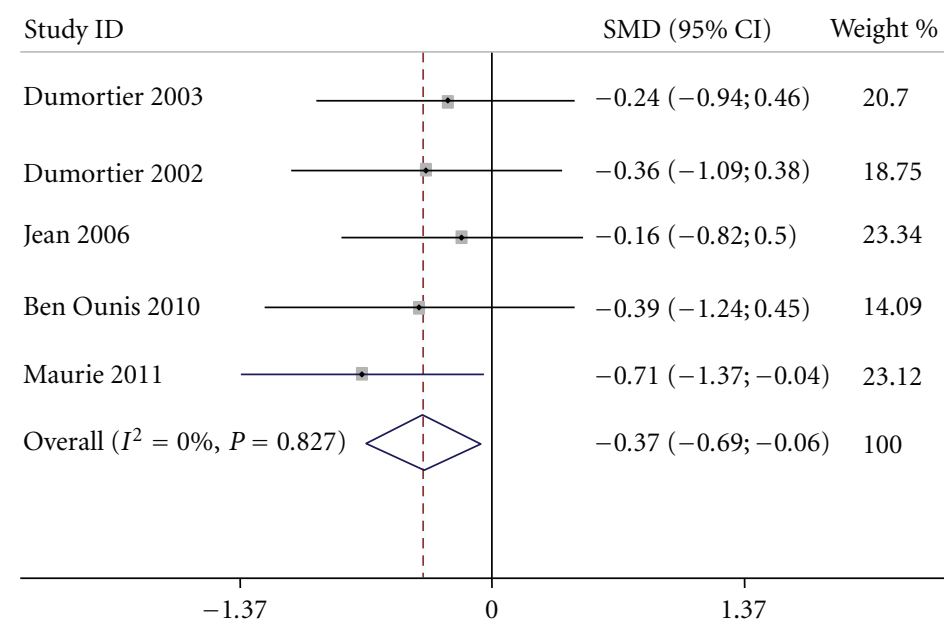

FIGURE 5: Pooled standardized mean difference and confidence intervals of weight comparing intervention versus control.

3.3.2. Fat Mass. Concerning this outcome, the metaregression showed that neither population $(P=0.19)$ nor intervention duration $(P=0.55)$ were significant moderators. Only nutrition remained significant $(<0.001)$.

Thus, separating population according to presence or not of nutritional intervention, the mean difference was significant for interventions that only considered exercise $(-1.49$ (95\% CI: $-2.04 ;-0.94))$, and was also larger for intervention including diet and exercise $(-8.32$ (95\% CI: $-10.89 ;-5.74)$ ). As for weight, no overlap was founded between the CI signifying a significant difference between the interventions. There was no heterogeneity in exercise interventions $\left(P=0.99, I^{2}=0.0 \%\right)$ as for diet + exercise $\left(P=0.10, I^{2}=48.6 \%\right)$.

3.3.3. Waist Circumference. The metaregression showed that population was not a significant moderator $(P=0.51)$, neither was intervention duration $(P=0.36)$. Only nutrition remained a significant moderator $(P=0.004)$ of waist circumference.

Thus, considering the presence of nutrition or not, the effect of exercise was still significant $(-3.51$ (95\% CI: -4.58 ; $-2.43))$ as diet + exercise $(-8.87(95 \%$ CI: $-11.98 ;-5.75))$ without significant difference due to the overlap between the CI. Then, no heterogeneity was noted either for exercise only $\left(P=0.98, I^{2}=0.0 \%\right)$ as for diet + exercise $\left(P=0.19, I^{2}=\right.$ $36 \%)$.

3.3.4. Cholesterol. As there was no heterogeneity in the meta-analysis $\left(P=0.02, I^{2}=52 \%\right)$, we did not analyze moderators of cholesterol.

3.3.5. Publication Bias. We looked for a publication bias for weight loss between before and after intervention using funnel plot representation (Figure 6). In absence of publication bias, the studies' results should be symmetrically placed about the line that represent the effect estimate to form a shape of "funnel," taking into account that the results from

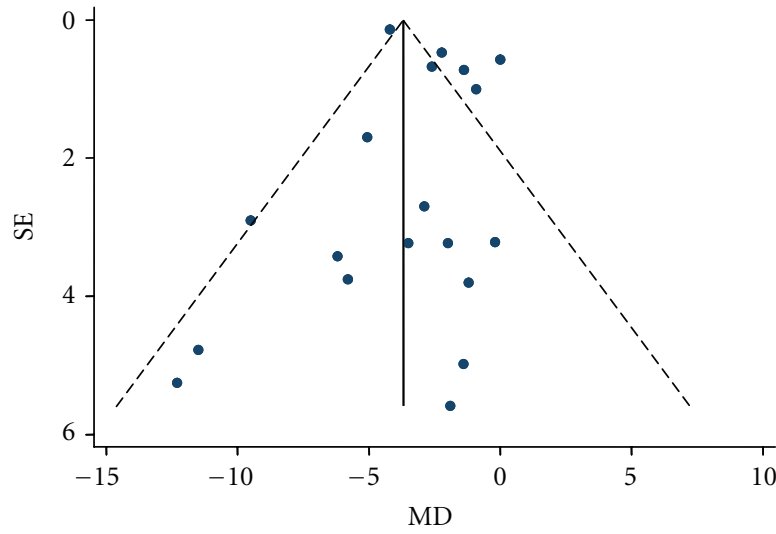

SE: standard error

MD: mean difference

Figure 6: Funnel plot of weight and its 95\% confidence interval in before to after design.

smaller the smaller studies would be more widely spread around the average effect because of their larger standard errors. On Figure 6, studies are equally spread around the average effect; however, we can observe a weak tendency for the smaller studies to give more important weight loss that the larger studies which can suggest a possibility of publication bias. It would signify that there are other small studies which have been carried out but which have not been published and that those included in this meta-analysis are biased in favor of weight loss. However, we cannot make the conclusion of the presence of a publication bias because this distribution may also arise from the small number of included studies.

\section{Discussion}

This meta-analysis confirms the conclusions of the individual studies, that are very low intensity training targeted at the 
level of maximal fat oxidation significantly decreases body weight, fat mass, waist circumference and total cholesterol. On the average, the effects of this variety of training are thus well confirmed, and their average magnitude is more precisely described.

Some methodological aspects of our meta-analysis need to be discussed. First, only 5 studies include a control (nonexercising) group. Actually, we found more studies testing the effect of this method versus caloric restriction group than versus a control nonexercising group. Although all the 5 studies including a control group evidenced a superiority of intervention versus control, the pooled estimate shows a medium size effect on weight loss $(-0.37)$ for the intervention group compared to the control group. This effect size could arise from a lack of power due to the low sample size: 128 intervention subjects versus 57 control subjects. However, if these results may seem low, they can be compared with the meta-analysis from $\mathrm{Wu}$ et al. [29]. In their meta-analysis, they compared the effect of diet + exercise versus diet only on weight loss and they obtained a standardized mean difference of -0.25 (95\% CI: -0.36 ; -0.14) in favor of diet + exercise whereas we obtained a standardized mean difference of -0.37 (95\% CI: $-0.69 ;-0.06)$ in favor of exercise intervention. These results showed that specific physical activity training at the level of maximal lipid oxidation could give results similar to those of other intervention even if these results should be interpreted carefully because the intervention was not identical.

The number of studies and especially of randomized controlled trials also requires comments. This number is quite small. Moreover, sample sizes in each study are relatively reduced, as they varied from 6 to 39 subjects. Therefore, there is a lack of large studies and especially of large randomized controlled trials for this variety of training. Another issue we have to discuss is the heterogeneity found in several analyses. Significant heterogeneity was detected for weight, fat mass and waist circumference comparing pre- and postintervention results. This heterogeneity could arise from the heterogeneity of included populations; indeed, participants suffer from various diseases: obesity [24], metabolic syndrome [16, 19], HIV-1 infection [17], T2D $[20,22,25,28]$, and psychiatric diseases [23]. In addition, participants present heterogeneous ages: some are adolescents, others are young adults and others are older adults. This source of heterogeneity was confirmed by the metaregression showing that the population was a significant moderator of the results and when we separated the analysis according to the population, the heterogeneity became nonsignificant. So, the absence of heterogeneity with significant results according to population reinforced the interest of training at maximal lipid oxidation throughout different populations with chronic diseases. However, population was only a significant moderator for weight. Another source of heterogeneity is the type of interventions that differed from a study to another. Indeed, several studies only proposed exercise to their intervention group whereas others included exercise as well as nutrition management. Furthermore, the analysis of metaregression showed that nutrition was a significant moderator for each studied outcome. We can observe that studies reported better results when diet is associated to exercise as evidenced by the $95 \%$ CI showing significant differences in weight and fat mass but not for waist circumference. The synergistic effect of diet and exercise in obesity is now well established [1], and metaanalyses show that exercise by its own improves the effect of diet [29]. However, the effect of diet and the effect of exercise are difficult to delineate and results from our meta-analysis concerning the nutrition should be interpreted in light of the fact that nutrition interventions were all hypocalorics.

Concerning the duration of interventions, it was not (as it could have been expected) a significant moderator. This could be explained by the fact that only the study from Fédou et al. [17] reported a duration superior to 3 months.

Interestingly, some studies [26] demonstrated an important average weight loss ( $8 \mathrm{~kg}$ over two months) with a protocol based on $90 \mathrm{~min} /$ day exercise at the level of maximal lipid oxidation. This could suggest that large weekly volumes of exercise training may be much more efficient than those used usually (i.e, $3 \times 45 \mathrm{~min} /$ week). Studies on the doseefficiency of this training procedure remain to be performed.

Actually, most of these studies used a moderate weekly amount of exercise (in most papers $135 \mathrm{~min}$ per week) according to the guidelines available in the early 2000s. It is interesting to point out that such a moderate training protocol has demonstrable metabolic effects, as evidenced by two biopsy studies $[20,30]$. An improvement in mitochondrial oxidation can be observed after only 2 months of training targeted on maximal lipid oxidation at only $90 \mathrm{~min} / \mathrm{wk}$, and is correlated to an increase in the ability to oxidize lipids at exercise [20,30-32]. This effect of training at the level of maximal lipid oxidation on the ability to oxidize lipids at exercise is demonstrated in all studies including this measurement. Therefore it is clear that the ability to oxidize lipids at exercise is increased by this kind of targeted training.

Whether it also modifies resting energy expenditure and resting lipid oxidation and make training more effective over $24 \mathrm{~h}$ remains to be studied.

The central questions about such protocols targeted at lipid oxidation levels are as the following: (1) does targeting training at this level of lipid oxidation improve results compared to more standard procedures or not; (2) is the energy deficit the only factor of the therapeutic effect of exercise or does targeting it on lipids make it more efficient? The debate is sometimes passionate but we think that both questions are not yet resolved and deserve careful consideration.

Personal targeting of exercise training is a classic issue in respiratory diseases so that some guidelines recommend it [33] on the basis of studies showing its superiority [34]. However other guidelines consider that there is no clear advantage of targeting and that standard procedures are efficient enough [35]. In heart or lung diseases the logic level for targeting is the ventilatory threshold $\left(V_{T}\right)$ because it is related to dyspnea which is a major symptom in these pathologies. Personalized targeting at the $V_{T}$ has also been proposed in diabetes [36] and proven a marked efficacy on the cost of diabetes treatment [37]. However, dyspnea being not a key symptom in obesity or diabetes, it was logic to 
propose a model of training based on a more metabolic parameter and the level of maximal lipid oxidation has been logically proposed for this purpose $[1,38,39]$.

Concerning obesity and exercise, there is literature saying that "fat loss depends on energy deficit only, independently of the method for weight loss" [40]. There is also a doseresponse relationship between the crude amount of exercise expressed in METs and the loss of abdominal fat [41]. However few recent studies suggest that the ability to oxidize lipids may explain the interindividual variability of the efficiency of exercise-based weight reduction procedures, so that individuals oxidizing more lipids at rest [42, 43] or during exercise $[44,45]$ respond better to exercise. Since exercise may have both orexigenic and satietogenic effects [46] (in trained and healthy participants), it can also be assumed that lipid oxidizing exercise is less orexigenic because it minimizes carbohydrate waste that occurs at higher intensities and may increase appetite. Accordingly, the weight-reducing effect of exercise targeted at the LIPOXmax may be mediated in part by alterations in food intake [47]. Although this issue remains conflictual, it is logic to investigate this question more thoroughly. At this time our meta-analysis of available data is unable to address this question.

Studies comparing training targeted on lipid oxidation with other training procedures are scarce. Comparison with aerobic interval training [28] suggests that the latter exhibits stronger effects on aerobic capacity, blood pressure, and blood lipids, while training targeted on lipids induces a greater fat loss and a better improvement of blood glucose levels in diabetics. There is also an interesting study that has not been included in this meta-analysis because it is not individually targeted at the LIPOXmax, but which aims at comparing endurance training at $40 \% \mathrm{VO}_{2} \max$ and $60 \%$ $\mathrm{VO}_{2}$ max. The level of $40 \% \mathrm{VO}_{2}$ max was chosen because it was expected to elicit a maximal level of lipid oxidation [48]. This study evidences a two-fold higher fat mass loss at $40 \%$ compared to $60 \% \mathrm{VO}_{2} \mathrm{max}$, and thus suggests that endurance training in the zone of lipid oxidation is more efficient than endurance at a higher power intensity to decrease fat mass. Clearly this issue requires more investigation.

If an interesting efficiency of exercise training targeted on lipid oxidation, as suggested by this meta-analysis, were further demonstrated, this method would appear rather attractive because the exercise test used for the targeting is easy to perform and does not require a maximal stress which is not always safe in obese or diabetic patients. In most countries a test before exercise training is required to ensure the safety of the procedure and is most of the time also used for targeting. In obese and/or diabetic subjects exercise calorimetry during a submaximal-graded exercise test could be one of the procedures employed for this purpose.

In conclusion, this meta-analysis shows that training targeted at maximal fat oxidation (mostly used 3 times a week) decreases fat mass and body weight and improves blood cholesterol. This method seems thus to be interesting in chronic diseases such as obesity and diabetes, but this evidence is based on small size studies and a host of issues remain to be clarified. Mostly, large-scale randomized controlled trials are needed to confirm these findings.

\section{Conflict of Interests}

There is no conflict of interests.

\section{Acknowledgments}

The authors wish to thank the University Hospital of Montpellier and the National Association of Research and Technology.

\section{References}

[1] D. R. Bensimhon, W. E. Kraus, and M. P. Donahue, "Obesity and physical activity: a review," American Heart Journal, vol. 151, no. 3, pp. 598-603, 2006.

[2] J. E. Donnelly, S. N. Blair, J. M. Jakicic, M. M. Manore, J. W. Rankin, and B. K. Smith, "Appropriate physical activity intervention strategies for weight loss and prevention of weight regain for adults," Medicine and Science in Sports and Exercise, vol. 41, no. 2, pp. 459-471, 2009.

[3] S. F. E. Praet and L. J. C. Van Loon, "Optimizing the therapeutic benefits of exercise in type 2 diabetes," Journal of Applied Physiology, vol. 103, no. 4, pp. 1113-1120, 2007.

[4] A. Pérez-Martin, M. Dumortier, E. Raynaud et al., "Balance of substrate oxidation during submaximal exercise in lean and obese people," Diabetes and Metabolism, vol. 27, no. 4, part 1, pp. 466-474, 2001.

[5] J. Achten, M. Gleeson, and A. E. Jeukendrup, "Determination of the exercise intensity that elicits maximal fat oxidation," Medicine and Science in Sports and Exercise, vol. 34, no. 1, pp. 92-97, 2002.

[6] D. P. van Aggel-Leijssen, W. H. Saris, A. J. Wagenmakers, G. B. Hul, and M. A. Van Baak, "The effect of low-intensity exercise training on fat metabolism of obese women," Obesity Research, vol. 9, no. 2, pp. 86-96, 2001.

[7] G. A. Brooks and J. Mercier, "Balance of carbohydrate and lipid utilization during exercise: the "crossover" concept," Journal of Applied Physiology, vol. 76, no. 6, pp. 2253-2261, 1994.

[8] J. M. Jakicic and A. D. Otto, "Treatment and prevention of obesity: what is the role of exercise?" Nutrition Reviews, vol. 64, no. 1, part 2, pp. S57-S61, 2006.

[9] J. M. Jakicic and A. D. Otto, "Physical activity considerations for the treatment and prevention of obesity," The American Journal of Clinical Nutrition, vol. 82, no. 1, supplement, pp. 226S-229S, 2005.

[10] J.-F. Brun, A.-J. Romain, and J. Mercier, "Maximal lipid oxidation during exercise (Lipoxmax): from physiological measurements to clinical applications. Facts and uncertainties," Science and Sports, vol. 26, no. 2, pp. 57-71, 2011.

[11] J. F. Brun, E. Varlet-Marie, A. J. Romain, and J. Mercier, "Measurement and physiological relevance of the maximal lipid oxidation rate during exercise (LIPOXmax)," in An International Perspective on Topics in Sports Medicine and Sports Injury, R. Kenneth Zaslav, Ed., InTech, 2012.

[12] M. Carayol, P. Grosclaude, and C. Delpierre, "Prospective studies of dietary alpha-linolenic acid intake and prostate cancer risk: a meta-analysis," Cancer Causes and Control, vol. 21, no. 3, pp. 347-355, 2010.

[13] O. Ben Ounis, M. Elloumi, I. Ben Chiekh et al., "Effects of twomonth physical-endurance and diet-restriction programmes on lipid profiles and insulin resistance in obese adolescent 
boys," Diabetes and Metabolism, vol. 34, no. 6, part 1, pp. 595600, 2008.

[14] F. Brandou, M. Dumortier, P. Garandeau, J. Mercier, and J. F. Brun, "Effects of a two-month rehabilitation program on substrate utilization during exercise in obese adolescents," Diabetes and Metabolism, vol. 29, no. 1, pp. 20-27, 2003.

[15] F. Brandou, A. M. Savy-Pacaux, J. Marie et al., "Impact of high- and low-intensity targeted exercise training on the type of substrate utilization in obese boys submitted to a hypocaloric diet," Diabetes and Metabolism, vol. 31, no. 4, part 1, pp. 327-335, 2005.

[16] M. Dumortier, F. Brandou, A. Perez-Martin, C. Fedou, J. Mercier, and J. F. Brun, "Low intensity endurance exercise targeted for lipid oxidation improves body composition and insulin sensitivity in patients with the metabolic syndrome," Diabetes and Metabolism, vol. 29, no. 5, pp. 509-518, 2003.

[17] C. Fédou, J. Fabre, V. Baillat et al., "Balance des substrats à l'exercice chez des patients infectés par le VIH 1 et présentant un syndrome lipodystrophique: effet d'un réentraînement ciblé par la calorimétrie d'effort," Science \& Sports, vol. 23, no. 3-4, pp. 189-192, 2008.

[18] O. Ben Ounis, M. Elloumi, M. Amri, Y. Trabelsi, G. Lac, and Z. Tabka, "Impact of training and hypocaloric diet on fat oxidation and body composition in obese adolescents," Science and Sports, vol. 24, no. 3-4, pp. 178-185, 2009.

[19] M. Dumortier, A. Pérez-Martin, E. Pierrisnard, J. Mercier, and J. F. Brun, "Regular exercise $(3 \times 45 \mathrm{~min} / \mathrm{wk})$ decreases plasma viscosity in sedentary obese, insulin resistant patients parallel to an improvement in fitness and a shift in substrate oxidation balance," Clinical Hemorheology and Microcirculation, vol. 26, no. 4, pp. 219-229, 2002.

[20] S. Bordenave, L. Metz, S. Flavier et al., "Training-induced improvement in lipid oxidation in type 2 diabetes mellitus is related to alterations in muscle mitochondrial activity. Effect of endurance training in type 2 diabetes," Diabetes and Metabolism, vol. 34, no. 2, pp. 162-168, 2008.

[21] O. Ben Ounis, M. Elloumi, G. Lac et al., "Two-month effects of individualized exercise training with or without caloric restriction on plasma adipocytokine levels in obese female adolescents," Annales d'Endocrinologie, vol. 70, no. 4, pp. 235241, 2009.

[22] E. Jean, E. Grubka, M. Karafiat et al., "P1-155—effets d'un entraînement en endurance ciblé par la calorimétrie à l'effort chez des diabétiques de type 2," Annales d'Endocrinologie, vol. 67, no. 5, p. 462, 2006.

[23] A.-J. Romain, J. Attal, A. Hermès et al., "Effects of endurance training targetd at the LIPOXmax in psychiatric patients treated by neuroleptics," Science and Sports, vol. 24, no. 5, pp. 265-268, 2009.

[24] M. C. Venables and A. E. Jeukendrup, "Endurance training and obesity: effect on substrate metabolism and insulin sensitivity," Medicine and Science in Sports and Exercise, vol. 40, no. 3, pp. 495-502, 2008.

[25] M. Mogensen, B. F. Vind, K. Højlund, H. Beck-Nielsen, and K. Sahlin, "Maximal lipid oxidation in patients with type 2 diabetes is normal and shows an adequate increase in response to aerobic training," Diabetes, Obesity and Metabolism, vol. 11, no. 9, pp. 874-883, 2009.

[26] M. Elloumi, O. Ben Ounis, E. Makni, E. Van Praagh, Z. Tabka, and G. Lac, "Effect of individualized weight-loss programmes on adiponectin, leptin and resistin levels in obese adolescent boys," Acta Paediatrica, International Journal of Paediatrics, vol. 98, no. 9, pp. 1487-1493, 2009.
[27] O. B. Ounis, M. Elloumi, E. Makni et al., "Exercise improves the ApoB/ApoA-I ratio, a marker of the metabolic syndrome in obese children," Acta Paediatrica, International Journal of Paediatrics, vol. 99, no. 11, pp. 1679-1685, 2010.

[28] J. Maurie, J.-F. Brun, E. Jean, A.-J. Romain, and J. Mercier, "Comparaison de deux modalités différentes d'activité physique (SWEET et Lipoxmax) chez des diabétiques de type 2," Science \& Sports, vol. 26, no. 2, pp. 92-96, 2011.

[29] T. Wu, X. Gao, M. Chen, and R. M. van Dam, "Longterm effectiveness of diet-plus-exercise interventions vs. dietonly interventions for weight loss: a meta-analysis: obesity management," Obesity Reviews, vol. 10, no. 3, pp. 313-323, 2009.

[30] K. Sahlin, M. Mogensen, M. Bagger, M. Fernström, and P. K. Pedersen, "The potential for mitochondrial fat oxidation in human skeletal muscle influences whole body fat oxidation during low-intensity exercise," American Journal of Physiology, vol. 292, no. 1, pp. E223-E230, 2007.

[31] I. Ara, S. Larsen, B. Stallknecht et al., "Normal mitochondrial function and increased fat oxidation capacity in leg and arm muscles in obese humans," International Journal of Obesity, vol. 35, no. 1, pp. 99-108, 2011.

[32] P. Nordby, B. Saltin, and J. W. Helge, "Whole-body fat oxidation determined by graded exercise and indirect calorimetry: a role for muscle oxidative capacity?" Scandinavian Journal of Medicine and Science in Sports, vol. 16, no. 3, pp. 209-214, 2006.

[33] "Guidelines for the rehabilitation of chronic obstructive pulmonary disease. French Language Society of Pneumology," Revue des Maladies Respiratoires, vol. 22, no. 5, part 3, pp. 7S87S14, 2005.

[34] G. Vallet, S. Ahmaïdi, I. Serres et al., "Comparison of two training programmes in chronic airway limitation patients: standardized versus individualized protocols," European Respiratory Journal, vol. 10, no. 1, pp. 114-122, 1997.

[35] C. L. Rochester, "Exercise training in chronic obstructive pulmonary disease," Journal of Rehabilitation Research and Development, vol. 40, no. 5, pp. 59-80, 2003.

[36] M. Kunitomi, K. Takahashi, J. Wada et al., "Re-evaluation of exercise prescription for Japanese type 2 diabetic patients by ventilatory threshold," Diabetes Research and Clinical Practice, vol. 50, no. 2, pp. 109-115, 2000.

[37] J.-F. Brun, S. Bordenave, J. Mercier, A. Jaussent, M. C. Picot, and C. Préfaut, "Cost-sparing effect of twice-weekly targeted endurance training in type 2 diabetics: a one-year controlled randomized trial," Diabetes and Metabolism, vol. 34, no. 3, pp. 258-265, 2008.

[38] M. A. van Baak, "Exercise training and substrate utilisation in obesity," International Journal of Obesity and Related Metabolic Disorders, vol. 23, supplement 3, pp. S11-S17, 1999.

[39] A. Pérez-Martin, E. Raynaud, and J. Mercier, "Insulin resistance and associated metabolic abnormalities in muscle: effects of exercise," Obesity Reviews, vol. 2, no. 1, pp. 47-59, 2001.

[40] B. Strasser, A. Spreitzer, and P. Haber, "Fat loss depends on energy deficit only, independently of the method for weight loss," Annals of Nutrition and Metabolism, vol. 51, no. 5, pp. 428-432, 2007.

[41] K. Ohkawara, S. Tanaka, M. Miyachi, K. Ishikawa-Takata, and I. Tabata, "A dose-response relation between aerobic exercise and visceral fat reduction: systematic review of clinical trials," International Journal of Obesity, vol. 31, no. 12, pp. 1786-1797, 2007. 
[42] N. D. Barwell, D. Malkova, M. Leggate, and J. M. R. Gill, "Individual responsiveness to exercise-induced fat loss is associated with change in resting substrate utilization," Metabolism, vol. 58, no. 9, pp. 1320-1328, 2009.

[43] M. Rosenkilde, P. Nordby, L. B. Nielsen, B. M. Stallknecht, and J. W. Helge, "Fat oxidation at rest predicts peak fat oxidation during exercise and metabolic phenotype in overweight men," International Journal of Obesity, vol. 34, no. 5, pp. 871-877, 2010.

[44] M. Hopkins, P. Caudwell, and C. Gibbons, "Fat oxidation during exercise is associated with medium-term exerciseinduce fat loss," Obesity Reviews, vol. 11, supplement 1, p. 183, 2010.

[45] P. Lavault, S. Deaux, A. J. Romain, C. Fédou, J. Mercier, and J. F. Brun, "Interest of quantification of muscle mass for interpreting exercise calorimetry," Science and Sports, vol. 26, no. 2, pp. 88-91, 2011.

[46] N. A. King, P. P. Caudwell, M. Hopkins, J. R. Stubbs, E. Naslund, and J. Blundell, "dual-process action of exercise on appetite control: increase in orexigenic drive but improvement in meal-induced satiety," American Journal of Clinical Nutrition, vol. 90, no. 4, pp. 921-927.

[47] J. F. Brun, D. Malatesta, and A. Sartorio, "Maximal lipid oxidationduring exercise: a target for individualizing endurance training in obesity and diabetes?" Journal of Endocrinological Investigation. In press.

[48] S. Lazzer, C. Lafortuna, C. Busti, R. Galli, F. Agosti, and A. Sartorio, "Effects of low- and high-intensity exercise training on body composition and substrate metabolism in obese adolescents," Journal of Endocrinological Investigation, vol. 34, no. 1, pp. 45-52, 2011. 


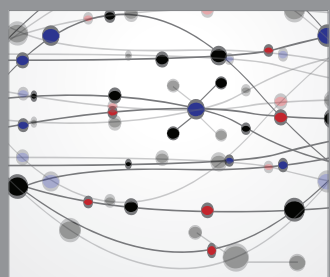

The Scientific World Journal
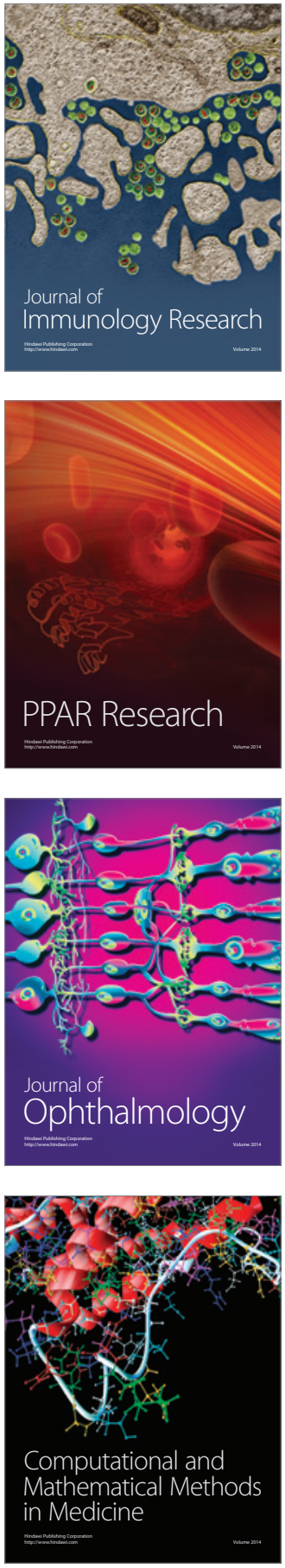

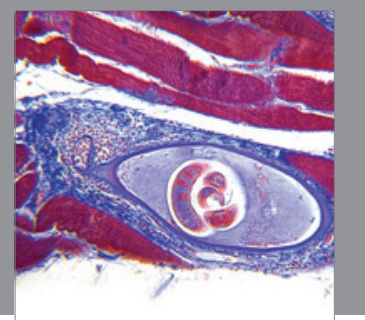

Gastroenterology

Research and Practice
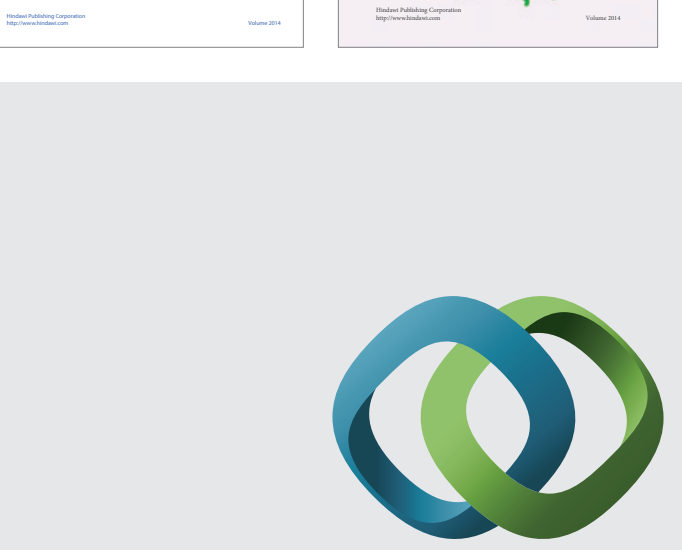

\section{Hindawi}

Submit your manuscripts at

http://www.hindawi.com
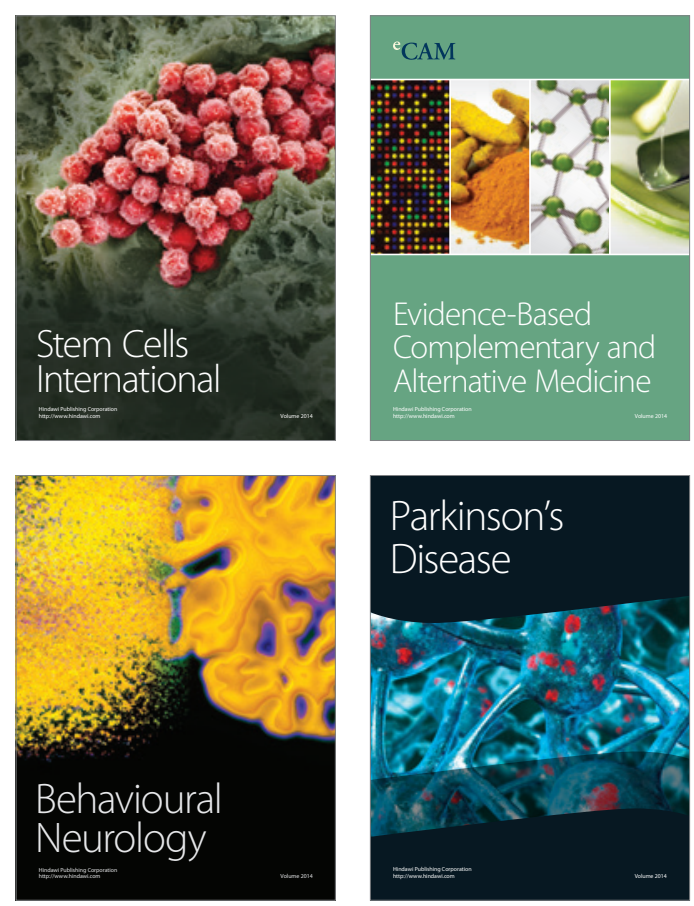

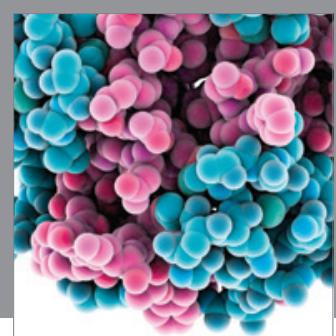

Journal of
Diabetes Research

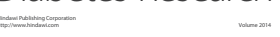

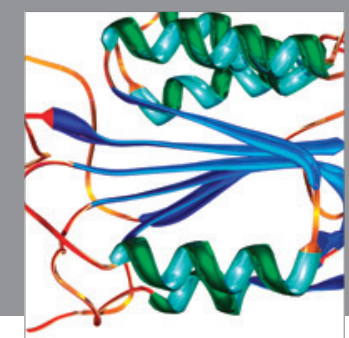

Disease Markers
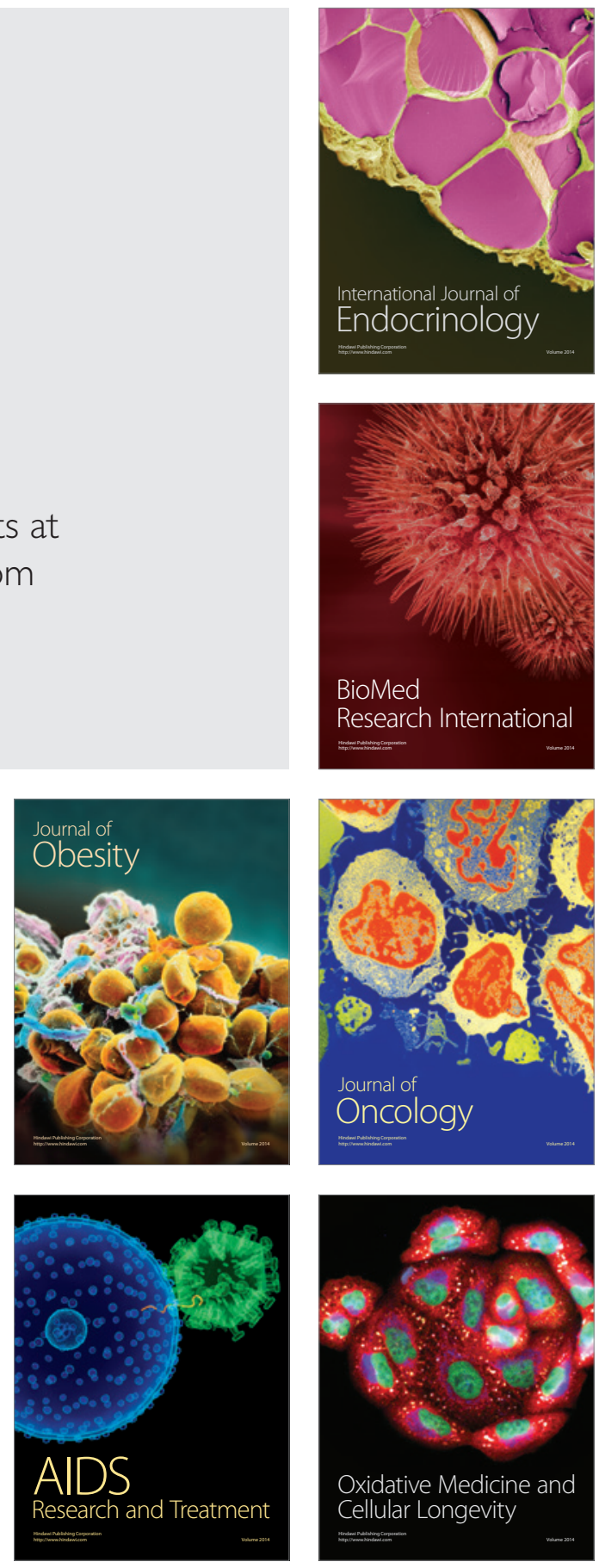